\title{
Review \\ Current and future technologies for breast cancer imaging
} James P Basilion

\begin{abstract}
Center for Molecular Imaging Research, NFCR Center for Molecular Analysis and Imaging, Massachusetts General Hospital-Harvard Medical School, Charlestown, Massachusetts, USA
\end{abstract}

Correspondence: James P Basilion, Assistant Professor, Center for Molecular Imaging Research, NFCR Center for Molecular Analysis and Imaging, Massachusetts General Hospital-Harvard Medical School, Building 149, $13^{\text {th }}$ Street \#5406, Charlestown, MA 02129, USA.

E-mail: basilion@helix.mgh.harvard.edu

Received: 17 November 2000

Accepted: 27 November 2000

Published: 13 December 2000
Breast Cancer Res 2001, 3:14-16

(C) Biomed Central Ltd on behalf of the copyright holder (Print ISSN 1465-5411; Online ISSN 1465-542X)

\section{Introduction}

Breast cancer is one of the most frequently diagnosed malignancies and the second leading cause of cancer deaths in American females [1]. Several improvements in diagnostic protocols have enhanced our ability for earlier detection of breast cancer, resulting in improvement of therapeutic outcome and an increased survival rate for breast cancer victims [2-4]. The importance of early detection of breast tumors therefore cannot be overemphasized.

Current early screening techniques are, however, neither comprehensive nor infallible, especially in women with radiographically dense breasts. Recent data from the Edinburgh randomized trial of breast screening as well as other retrospective analyses of 'false-negative' mammograms demonstrate that approximately one-third of diagnosed cancers were visible on early 'negative' mammograms $[2,5,6]$. Early detection of breast cancer therefore still presents a significant diagnostic challenge. Imaging techniques that improve breast cancer detection, localization, and evaluation of therapy would be a major breakthrough in combating the disease.

Detection of malignant tumor cells in a background of normal or hyperplastic benign tissue is often based on differences in physical properties between tissues, which are frequently minimal (eg proton relaxation times, X-ray absorption, ultrasound scattering), resulting in low contrast resolution; that is, small tumors are not detectable. To enhance detection of small tumors, several groups have been developing the concept of molecular imaging as a way to increase the signal to noise ratio by detecting the differences in 'molecular properties' between cancer and normal tissues that arise as a result of malignant transformation. This should, in theory, allow for earlier detection of smaller tumors.

The following reviews in this series will describe how state-of-the-art imaging modalities (magnetic resonance [MR] imaging, magnetic resonance spectroscopy, nuclear imaging, and optical imaging) are being used to more precisely identify human breast tumors and monitor chemotherapeutic response. Not all of the cited technologies are yet able to take advantage of the underlying molecular properties resulting in altered tumor biology; however, many have been developed to the point of being able to exploit macromolecular changes in tumor physiology and/or metabolism to increase detection of diseased tissue. Part of the problem in exploiting the underlying molecular changes manifest as altered physiology or metabolism is the lack of genetic information. As advances in gene profiling allow dissection of underlying molecular profiles of tumors, imaging approaches will be more molecularly based. Uncovering and understanding the molecular aberrations directly underlying the measurable biological differences, as examples from our own work demonstrate (see below), will allow molecular refinement of the imaging tools and, presumably, more sensitive tumor detection.

\section{Molecular imaging of cancer}

Molecular imaging, defined as the in vivo characterization and measurement of biological processes at the cellular and molecular level, is an attempt to image the molecular 


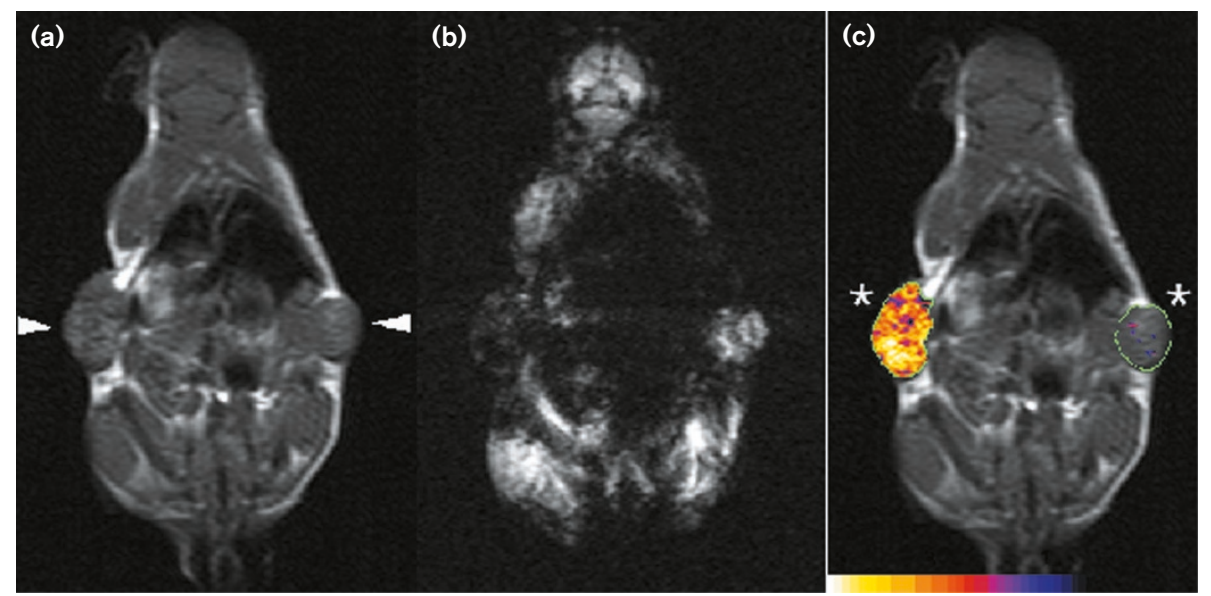

In vivo MR imaging. MR image of a single mouse with TfR+ and TfR- flank tumors. (a) The $\mathrm{T}_{1}$-weighted coronal SE image (imaging time, 3.5 min; 300 $\times 300 \times 3000 \mu \mathrm{m}^{3}$ voxel resolution). TfR- tumors (right arrowhead) and TfR+ tumors (left arrowhead) have similar signal intensity. (b) Corresponding $\mathrm{T}_{2}$-weighted gradient echo image showing significant difference between TfR- and TfR+tumors (imaging time, $8 \mathrm{~min} ; 300 \times 300 \times 3000 \mu \mathrm{m}^{3} \mathrm{voxel}$ resolution). TfR-mediated cellular accumulation of the superparamagnetic probe decreases signal intensity as expected. These differences in MR signal intensity were most pronounced using $\mathrm{T}_{2}$ - and $\mathrm{T}_{2}{ }^{*}$-weighted imaging pulse sequences consistent with the increased $\mathrm{R} 2$ upon cellular internalization. (c) Composite of a $\mathrm{T}_{1}$-weighted spin echo image obtained for anatomic detail with superimposed R2 changes after Tf-MION administration displayed in a color map. Note the difference in R2 changes between the TfR+ and TfR- tumors (asterisks). Scale bar, $10 \mathrm{~mm} ; N=1$. (Reproduced with permission from Weissleder et al [13].)

make-up of the macrofeatures currently visualized using 'classical' diagnostic imaging modalities. Differences in molecular properties of cells may result from genetic manipulation via gene transfer vectors or, as is the case for cancer, are manifest as a result of inappropriate expression of specific gene products in the diseased tissue. While the expression levels of many genes may be altered in cancer, there are few examples where an overexpressed gene is detectable in the cancer tissue but not in normal tissue. To exploit differences in the genetic make-up of normal and malignant tissues, it is therefore necessary to develop an imaging strategy that detects relative differences in gene expression between these tissues.

The molecular imaging approach we have been developing is based on the discovery that several cell-surface internalizing receptors are overexpressed on cancer cells relative to surrounding tissues [7]. Several studies of different cancers, including cancers of the breast, have correlated expression of the transferrin receptor (TfR) to tumor grade and metastatic potential, and it has been suggested that receptor levels may be helpful in grading tumors and determining prognosis [8-10]. The transferrin receptor is a cell-surface internalizing receptor responsible for almost all iron sequestration in mammalian cells. Overexpression of the TfR on cancer cells is presumably secondary to increased cellular metabolism, for which iron is required. We are attempting to enhance detection of tumor tissues by trying to directly image this altered molecular property of cancers using MRI.
We have hypothesized that conjugating ligands for the TfR to a MR contrast agent (superparamagnetic monocrystalline ironoxide nanoparticles [MIONs]) would selectively increase uptake of MIONs into cells overexpressing the receptor, resulting in an altered MR signal. This hypothesis has been tested using the TfR and holo transferrin-MION (Tf-MION) conjugates. Both other workers and ourselves have shown that as little as a fivefold [11-13] overexpression of the TfR on tumor cells can be detected using TfMION, which results in a 400-fold increase in MR imaging sensitivity in vivo (Fig. 1). Detection of a modest fivefold increase in the TfR receptor depends on several synergistic factors characteristic of the receptor system (detailed in [13]). These characteristics are shared by several other receptors, suggesting that this concept for increasing MR imaging sensitivity could be extended to other receptor systems and is applicable to a large number of cancers, including breast cancer. Application of molecular imaging to breast cancer imaging has the potential to radically improve breast cancer detection, localization, and evaluation of therapy, and would be a major breakthrough in combating the disease.

Studies aimed at determining the level of overexpression of the transferrin and other internalizing cell surface receptors on human breast cancers are currently underway in our laboratory. The statistically significant database of receptor targets on breast cancers that will be created by these studies will enable probe development to allow molecular imaging based on tumor-selective markers. 
Demonstrating the feasibility of enhanced MR imaging of cancer due to overexpression of cell surface receptors can, in theory, be applied to other biological systems. For example, targeted MR imaging agents might be useful to enhance MR imaging of tumor vascularization. It is clear in Dr Leach's review on MR imaging of angiogenesis that differences in dynamic contrast measurements can aid in discerning normal or benign from neoplastic breast tissues. The molecular basis for these detectable differences may be explained, in part, by the findings of Croix et al showing that dramatic differences in endothelial cell gene expression (20-fold and greater) exist between endothelial cells derived from tumor and nontumor related vasculature [14]. Development of imaging agents that would target these relative differences in gene expression could significantly enhance the sensitivity of MR imaging to detect malignancy-related angiogenesis. This approach is, of course, not limited to MR techniques or angiogenesis imaging. Similarly, identification of cancer-related gene products (eg enzymes) whose overexpression in tumors themselves or tumor vasculature could be exploited to activate MR, optical, or nuclear imaging probes (see $[15,16]$ ) would be a significant addition to the cutting edge technologies described in the subsequent reviews.

Only now is the potential of molecular imaging being fully recognized. The progression of imaging sciences and molecular analysis of the human organism have traveled on parallel paths - only recently to intersect and inform insightful developments for molecular imaging. In the following sections, the authors review the current state of several imaging modalities as they apply to breast cancer and will highlight where interdisciplinary nodes occur by referring to or describing studies that are beginning to exploit these intersections to develop novel molecular imaging strategies.

\section{References}

1. Goldsmith MF: Leading sites of new cancer cases and deaths - 1999 estimates [letter]. JAMA 1999, 281:405.

2. Alexander FE, Anderson TJ, Brown HK, Forrest AP, Hepburn W, Kirkpatrick AE, Muir BB, Prescott RJ, Smith A: 14 years of follow-up from the Edinburgh randomised trial of breastcancer screening [see comments]. Lancet 1999, 353: 1903-1908.

3. Haffty BG, Lee C, Philpotts L, Horvath L, Ward B, McKhann C, Tocino I: Prognostic significance of mammographic detection in a cohort of conservatively treated breast cancer patients [see comments]. Cancer J Sci Am 1998, 4:35-40.

4. Moller P, Reis MM, Evans G, Vasen H, Haites N, Anderson E, Steel CM, Apold J, Lalloo F, Maehle L, Preece P, Gregory H, Heimdal K: Efficacy of early diagnosis and treatment in women with a family history of breast cancer. European Familial Breast Cancer Collaborative Group. Dis Markers 1999, 15: 179-186.

5. Saarenmaa I, Salminen T, Geiger U, Holli K, Isola J, Karkkainen A, Pakkanen J, Piironen A, Salo A, Hakama M: The visibility of cancer on earlier mammograms in a population-based screening programme. Eur J Cancer 1999, 35:1118-1122.

6. Patel MR, Whitman GJ: Negative mammograms in symptomatic patients with breast cancer. Acad Radiol 1998, 5:26-33.
7. White S, Taetle R, Seligman PA, Rutherford M, Trowbridge IS: Combinations of anti-transferrin receptor monoclonal antibodies inhibit human tumor cell growth in vitro and in vivo: evidence for synergistic antiproliferative effects. Cancer Res 1990, 50:6295-6301.

8. Basar I, Ayhan A, Bircan K, Ergen A, Tasar C: Transferrin receptor activity as a marker in transitional cell carcinoma of the bladder. Br J Urol 1991, 67:165-168.

9. Recht L, Torres CO, Smith TW, Raso V, Griffin TW: Transferrin receptor in normal and neoplastic brain tissue: implications for brain-tumor immunotherapy [see comments]. J Neurosurg 1990, 72:941-945.

10. Inoue T, Cavanaugh PG, Steck PA, Brunner N, Nicolson GL: Differences in transferrin response and numbers of transferrin receptors in rat and human mammary carcinoma lines of different metastatic potentials. J Cell Physiol 1993, 156:212-217.

11. Kresse MS, Wagner S, Pfefferer D, Lawaczeck R, Elste V, Semmler W: Targeting of ultrasmall superparamagnetic iron oxide (USPIO) particles to tumor cells in vivo by using transferrin receptor pathways. Magn Reson Med 1998, 40:236-242.

12. Moore A, Basilion JP, Chiocca EA, Weissleder R: Measuring transferrin receptor gene expression by NMR imaging. Biochim Biophys Acta 1998, 1402:239-249.

13. Weissleder R, Moore A, Mahmood U, Bhorade R, Benveniste $H$, Chiocca EA, Basilion JP: In vivo magnetic resonance imaging of transgene expression. Nat Med 2000, 6:351-355.

14. St Croix B, Rago C, Velculescu V, Traverso G, Romans KE, Montgomery E, Lal A, Riggins GJ, Lengauer C, Vogelstein B, Kinzler KW: Genes expressed in human tumor endothelium. Science 2000, 289:1197-1202.

15. Weissleder $\mathrm{R}$, Tung $\mathrm{CH}$, Mahmood $\mathrm{U}$, Bogdanov A Jr: In vivo imaging of tumors with protease-activated near-infrared fluorescent probes. Nat Biotechnol 1999, 17:375-378.

16. Louie AY, Huber MM, Ahrens ET, Rothbacher U, Moats R, Jacobs $\mathrm{RE}$, Fraser SE, Meade TJ: In vivo visualization of gene expression using magnetic resonance imaging. Nat Biotechnol Markers 2000, 18:321-325. 